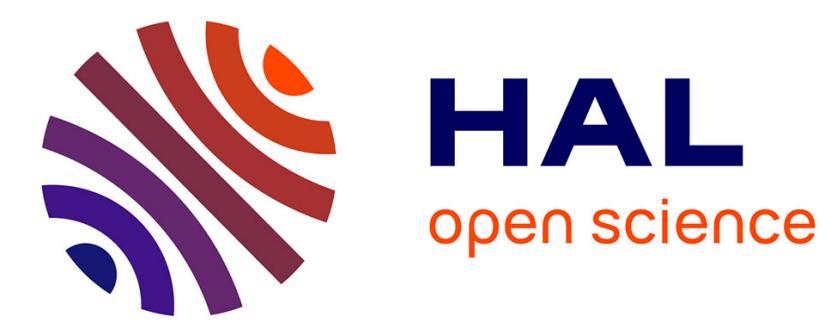

\title{
Dynamic Damage Law with Fragmentation Length: Strain-Rate Sensitivity of the Tensile Response \\ Cristian Dascalu
}

\section{To cite this version:}

Cristian Dascalu. Dynamic Damage Law with Fragmentation Length: Strain-Rate Sensitivity of the Tensile Response. Journal of Dynamic Behavior of Materials, 2021, 7 (1), pp.156-160. 10.1007/s40870020-00262-8 . hal-02915356

\section{HAL Id: hal-02915356 \\ https://hal.univ-lorraine.fr/hal-02915356}

Submitted on 14 Aug 2020

HAL is a multi-disciplinary open access archive for the deposit and dissemination of scientific research documents, whether they are published or not. The documents may come from teaching and research institutions in France or abroad, or from public or private research centers.
L'archive ouverte pluridisciplinaire HAL, est destinée au dépôt et à la diffusion de documents scientifiques de niveau recherche, publiés ou non, émanant des établissements d'enseignement et de recherche français ou étrangers, des laboratoires publics ou privés. 


\title{
Dynamic damage law with fragmentation length : strain-rate sensitivity of the tensile response
}

\author{
Cristian Dascalu
}

Received : date / Accepted : date

Résumé A damage law for dynamic failure in brittle solids is proposed in the present note. The new evolution equation is obtained by incorporating the GradyGlenn-Chudnovsky average fragment size expression as the microstructural length of a two-scale damage model. In this construction, the internal length of the model explicitly depends on the local strain rate and the material parameters. The capacity of the new approach to account for the strain-rate sensitivity of the tensile strength is illustrated by comparison of the predicted material and structural responses with experimental data for concrete and ceramic materials.

Keywords Dynamic damage law · Microstructural fragmentation length · Rate-sensitivity of tensile strength $\cdot$ Concrete $\cdot$ Alumina ceramic

\section{Introduction}

The dynamic fragmentation of brittle materials is a complex failure process involving nucleation, growth and coalescence of microcracks leading to the breaking of the solid body into many fragments. The size of the resulting fragments is essentially given by the mutual distance between neighbor microcracks which, by coalescence, induce the detachment of the matter. Such a microscopic length, characterizing the spatial distribution microcracks and the size of the final fragments, not only depends on the material properties of the solid but also on the strain rate.

\section{Dascalu}

Université de Lorraine, CNRS, Arts et Métiers ParisTech, LEM3, F-57000 Metz, France

E-mail: cristian.dascalu@univ-lorraine.fr
Based on energy analysis and assuming that the kinetic energy is fully converted to fracture energy, Grady [10] formulated a high strain-rate law for the average fragment size. By including the contribution of the elastic energy, dominant at low strain rates, Glenn and Chudnovsky [9] improved the Grady's model. This latter formulation is adopted in the present contribution as the Grady-Glenn-Chudnowsky (GGC) fragment size.

In the last decades, many works have been dedicated to dynamic brittle failure of solids and the associated microstructural aspects like the contributions in Levy and Molinari [15], Daphalapurkar et al. [2], Drugan [5], Zhou et al. [19], Grady [11], Kimberley et al. [14], Hu et al. [12]. A method for deriving damage equations from small-scale fracture criteria for microcracks has been proposed $[13,3]$ for dynamic evolutions. This approach relying on the asymptotic homogenization technique allows for the presence of a microstructural length, representing the size of the solid segment between neighbor microcracks, in the macroscopic damage law. The model has been used to simulate rapid failure in brittle polymers, ceramics or concrete materials in $[13,3,1,4]$. In these computations, the microstructural length has been assumed to be constant and it was calibrated for the specific range of strain-rates of the simulated experiment. In the present contribution, we will use the strain-rate dependent fragment size expression GGC as the microstructural length of the model.

The tensile response predicted by the new damage law will be analyzed in connection with experimental results for concrete and ceramic materials. We will focus on the strain-rate sensitivity of the tensile strength as reported for the direct tension and spalling tests on concrete $[6,7]$ and the splitting and spalling tests for alumina ceramics $[16,8,20]$. 
The note is organized as follows. In section 2, the microstructural arguments and the general formulation of the new model are given. The predicted strain-rate sensitivity of the tensile strength is analyzed in Section 3 for the local material response and in Section 4 for the structural simulations of the spalling test.

\section{General damage equations}

The framework of the two-scale developments [13,4] is that of a solid containing a locally periodic distribution of microcracks, as illustrated in Figure 1. The microstructural length $l_{c}$ is the mutual distance between neighbor microcracks and also the size of the periodic cells. It is supposed to be much smaller than a characteristic length $L_{c}$ of the macroscopic structure. The microcracks evolve in mode $\mathrm{I}$, their length is denoted by $l(t)$ and the macroscopic damage parameter is defined as the normalized crack length $d=\frac{l}{l_{c}}$. Local periodicity means slow small-scale variations of $l$, such that in a vicinity of a macroscopic point the distribution may be considered as periodic, of period $l_{c}$.

Use of he asymptotic homogenization method $[13$, 4] leads to the equations of motion

$\rho \frac{\partial^{2} u_{i}}{\partial t^{2}}=\frac{\partial \sigma_{i j}}{\partial x_{j}}$

and the stress-strain constitutive laws

$\sigma_{i j}=C_{i j k l}(d) e_{k l}$

for the macroscopic fields of displacement $u_{i}\left(x_{j}, t\right)$, strain $e_{k l}\left(x_{j}, t\right)=\frac{1}{2}\left(\frac{\partial u_{k}}{\partial x_{l}}+\frac{\partial u_{l}}{\partial x_{k}}\right)$ and damage $d\left(x_{j}, t\right)$, with the effective coefficients $C_{i j k l}$ depending on the damage variable $d$ and with the mass density $\rho$.

The dynamic evolution of damage is described by the law $[13,3,4]$ :

$\frac{d d}{d t}=\frac{2 C_{R}}{l_{c}}\left\langle 1-\frac{\mathcal{G}_{c}}{l_{c} \mathcal{Y}}\right\rangle$

where $\langle$.$\rangle represents the positive part. Here \mathcal{G}_{c}$ the fracture energy of the material, $C_{R}$ is the Rayleigh wave speed and $\mathcal{Y}=-\frac{1}{2} \frac{d C_{i j k l}(d)}{d d} e_{k l} e_{i j}$ is the damage energyrelease rate. We remark that the microstructural length $l_{c}$ is present in the equation (3). This damage law has been obtained from a Griffith-type propagation criterion with the microscopic kinetic energy present in the expression of the energy release rate $[13,3]$.

In previous works $[13,4]$ the coefficients $C_{i j k l}(d)$ were obtained by computations on the unit cell containing a single crack and with periodic boundary conditions. A particular orientation of microcracks induces anisotropy

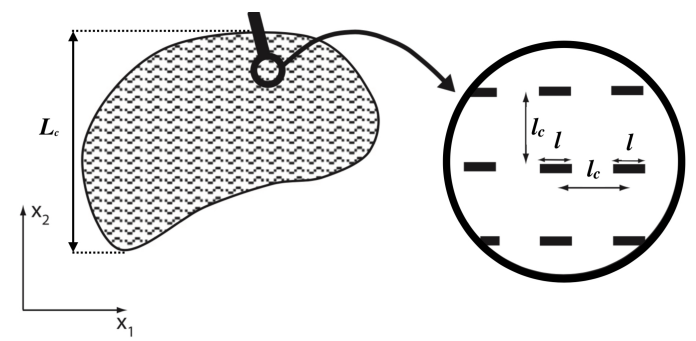

Figure 1 Micro-fissured medium with locally periodic microstructure, where $l_{c}$ is the size of a period, $l$ is the local micro-crack length and $L_{c}$ is a characteristic length of the macroscopic structure.

in the macroscopic response. Since in many physical situations the specimens can be considered as macroscopically isotropic, with families of microcracks developing in different directions at the small scale, in what follows we adopt the expressions $[3,1]$ :

$C_{i j k l}(d)=(1-d)^{a} c_{i j k l}$

where $c_{i j k l}$ are the elastic coefficients of the virgin material, assumed to be homogeneous and isotropic. The parameter $a>0$ has been considered in $[17,1]$ to induce a realistic softening response for quasi-brittle materials like concrete. Its effect on the local model will be studied in the next section. For $a \leq 1$ the resulting behavior is highly brittle, while for $1<a \leq 2$ a quasi-brittle response is obtained.

The use of the elasto-damage system (1-4) to describe rapid failure processes in brittle materials requires the choice of the microstructural length $l_{c}$. In the simulations performed for different brittle materials in Keita et al. [13], Dascalu [3], Atiezo et al. [1], Dascalu and Gbetchi [4], we assumed that $l_{c}$ is a constant. Its choice depends not only on the size of the microstructure but also on the specific range of strain-rates involved. The distribution of microcracks in real materials depend on the loading rate and the choice of a constant $l_{c}$ could be reasonable for a limited interval of values. To cover a large domain of strain rates, we need to continuously adapt the microstructural length.

Since $l_{c}$ represents the physical distance between neighbor microcracks, as shown in Figure 1, and since families of microcracks of different orientations are allowed to coexist at the small scale, it is natural to associate the microstructural length of the model to the fragment size of dynamic fragmentation processes.

Inspired by the fragmentation models existing in the literature, a general form of the strain-rate dependence for $l_{c}$ can be written as :

$l_{c}=f\left(\left\langle\dot{e}_{v}\right\rangle\right)$ 


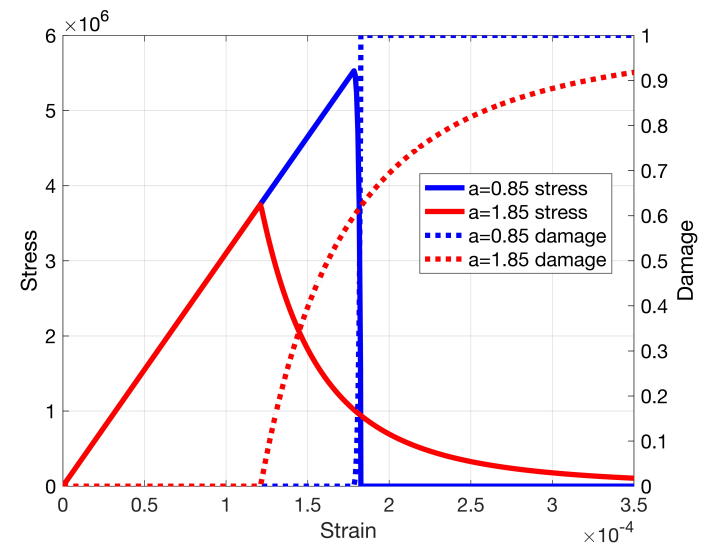

Figure 2 Local stress and damage response for two values of the parameter $a=0.85$ and $a=1.85$.

where $\left\langle\dot{e}_{v}\right\rangle$ is the expansive volumetric strain rate, with $e_{v}=e_{k k} / 3$ and $f$ a decreasing function specific to the adopted fragment size law. To illustrate the general approach, in the present note the expression of $f$ proposed by Glenn and Chudnovsky [9] will be considered.

The expression (5) of $l_{c}$ should be used in (3) to obtain the damage law of the new model and the elastic and damage fields are obtained by solving the resulting coupled system (1-5).

\section{Local response analysis}

Energy balance arguments concerning to the conversion of the kinetic energy into fracture energy led Grady [10] to formulate a law for the average fragment size, valid for high strain rates. By including the contribution of the elastic energy, relevant at low strain rates, Glenn and Chudnovsky [9] improved Grady's model. This last expression is used in what follows.

The average fragment size is given in [9] :

$l_{c}=4 \sqrt{\frac{\alpha}{3}} \sinh \left(\frac{\phi}{3}\right)$

with

$\phi=\sinh ^{-1}\left[\beta\left(\frac{3}{\alpha}\right)^{3 / 2}\right] ; \alpha=\frac{3 \sigma_{c}{ }^{2}}{\rho E \dot{e}^{2}} ; \beta=\frac{3 \mathcal{G}_{c}}{2 \rho \dot{e}^{2}}$

where $\dot{e}$ is the one-dimensional strain rate, $\sigma_{c}$ is the tensile strength and $E$ the Young modulus.

The expression of $l_{c}$ should be used in the damage law (3). In the 1D setting, the stress-strain law takes the form $\sigma=(1-d)^{a} E e$ and the damage energy-release rate is $\mathcal{Y}=\frac{a}{2}(1-d)^{a-1} E e^{2}$.

To investigate the local response predicted by the new model, we consider a strain history $e(t)=\dot{e} . t$, with constant strain rate $\dot{e}$. For a given $\dot{e}$, the new damage equation is solved numerically using a forward Euler finite-difference scheme. The computed damage solution $d(t)$ is then replaced in the stress-strain law to get the stress evolution $\sigma(t)$. In this way, the predicted tensile strength is obtained for different strain rate values.

Consider first the case of concrete material. The material constants of R30A7 concrete [6,7] are $E=$ $31 \mathrm{GPa}, \rho=2200 \mathrm{~kg} / \mathrm{m}^{3}, \mathcal{G}_{c}=60 \mathrm{~J} / \mathrm{m}^{2}$ and the quasistatic strength $\sigma_{q s}=3.6 \mathrm{MPa}$. The choice of the parameter $a$ can be done by calibration of the predicted stress-strain curve for a particular strain-rate. In Figure 2 such stress and damage response is given for two values of the parameter $a=0.85$ and $a=1.85$, at strain rate $\dot{e}=0.01 \mathrm{~s}^{-1}$ and the previous material parameters. We note the very brittle behavior for $a=0.85$ and the quasi-brittle softening for $a=1.85$. This latter value is retained for concrete.

We remark that for $a=1$ the use of the relations (6-7) with $\sigma_{c}=\sigma_{q s}$ in the damage model predicts exactly the tensile strength $\sigma_{q s}$ for the quasi-static regime. When $a \neq 1$, in order to retrieve the quasi-static tensile strength with the damage predictions we need to choose a different input value $\sigma_{c}$ in (6-7). For example, in the case $a=1.85$, taking $\sigma_{c}=5.094 M P a$ we retrieve the strength $\sigma_{q s}=3.6 \mathrm{MPa}$ by solving the damage equation in the quasi-static regime. This adjustment of the fragment size expression, for $a \neq 1$, guarantees the physical strength prediction for low strain rates.

In Figure 3 the computed tensile strength for different strain rates is compared to the experimental values obtained in direct tension tests [6] and spalling tests [7]. Two model curves are presented, corresponding to quasi-static and low-dynamic calibrations. For

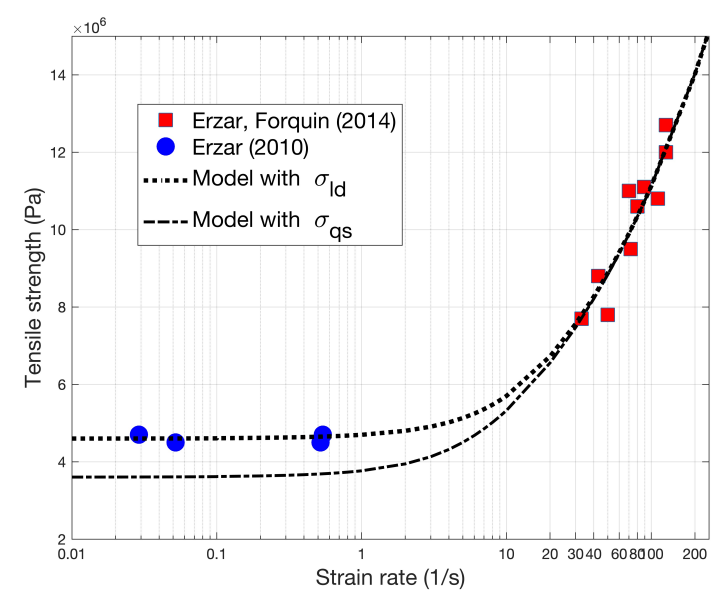

Figure 3 Model predictions with quasi-static and lowdynamic calibrations and experimental values $[6,7]$ of tensile strength vs. strain rate for R30A7 concrete. 
the first one, the above-described procedure for the choice of $\sigma_{c}$ has been used to obtain the strength $\sigma_{q s}$ in the quasi-static regime. For the second one, $\sigma_{c}=$ 6.255 $\mathrm{MPa}$ allows to retrieve the strength $\sigma_{l d}=4.6$ $M P a$ at the strain rate $0.0405 s^{-1}$. This represents the mean value of the lowest two experimental strengths reported in [6] for the direct tension tests.

We note the good agreement between experiments and simulations with the low-dynamics calibration. For high strain rates the two model curves are identical and fit well with the measured values. In the low-dynamics regime, the $\sigma_{l d}$ curve perfectly fits the second group of experimental strengths, obtained for strain rates close to $0.5 \mathrm{~s}^{-1}$, while the $\sigma_{q s}$ calibration underestimates the strength values. Only the $\sigma_{l d}$ predictions cover the full dynamic range of tensile strengths.

When approaching the quasi-static strain-rates, it is known that the fragment size (6) is relatively constant (e.g. [19] for comparison with other laws) and the corresponding strengths calculated with the damage law are not decreasing enough to reach $\sigma_{q s}$. Further developments of the present model may include the use of a different fragment size expression exhibiting more important variations at low rates, like the one proposed by Zhou et al. [19].

A second example concerns the alumina ceramics. The material properties [20] are $E=360 G P a, \rho=$ $3850 \mathrm{~kg} / \mathrm{m}^{3}, \sigma_{q s}=202.8 \mathrm{MPa}, \mathcal{G}_{c}=40 \mathrm{~J} / \mathrm{m}^{2}$. Since the fracture energy is not given in [20], an intermediate value among those existing in the literature $[18,16]$ has been chosen. Corresponding to the very brittle behavior of the ceramic material, similar to the case $a<1$ in Figure 2, the value $a=0.4$ has been chosen.

We compute the solutions of the damage problem for quasi-static and low-dynamic calibrations. The first one is obtained with the input stress $\sigma_{c}=129.8 \mathrm{MPa}$, while for the second one we choose $\sigma_{c}=152.85 \mathrm{MPa}$ in the fragment size law (6-7). The resulting strengths are represented in Figure 4 together with the experimental values obtained for high-purity $(\geq 99.5 \%)$ alumina ceramics in the splitting (Brazilian) and the spalling tests reported by Galvez Diaz-Rubio et al. [8] and the shockless spalling tests by Zinszner et al. [20].

The low-dynamics calibration of the model is done with the strength measured in the Brazilian test [8] : $\sigma_{l d}=243 \mathrm{MPa}$ for a strain rate of $76 \mathrm{~s}^{-1}$. As shown in Figure 4 , the $\sigma_{l d}$ prediction is in relatively good agreement with the strength measured in the spalling test [8] if we take into account the reported standard deviation. It is also in very good agreement with the experimental values reported in [20] for higher strain rates. As in the case of concrete, the model predictions for the tensile strength cover the full dynamics range.

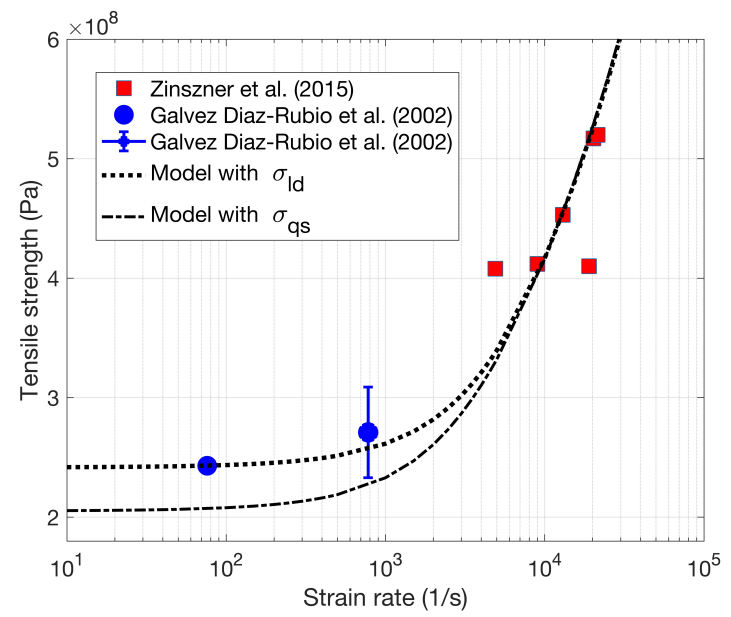

Figure 4 Model predictions with $\sigma_{q s}$ and $\sigma_{l d}$ and experimental tensile strengths for alumina ceramics, measured in the splitting and the spalling tests by Galvez Diaz-Rubio et al. [8] and the shockless spalling tests by Zinszner et al. [20].

Represented in Figure 4 is also the $\sigma_{q s}$ calibration which provides correct predictions for high strain-rates but underestimates the strength in the low dynamics regime. As explained previously, further extensions of the model to recover the quasi-static domain may involve the integration of a different fragment size expression, but this is beyond the objective of the present note.

We also note the necessity to adapt the model when the strain rates enter a regime where equilibrium fragmentation is no longer a reasonable assumption. Grady [11] noted such limits for brittle failure at strain rates of the order $10^{5} \mathrm{~s}^{-1}-10^{6} \mathrm{~s}^{-1}$ in glass and ceramic materials. He also proposed some line of extending the existing results to non-equilibrium brittle fragmentation but such a theory is still in development.

\section{Spalling test for concrete}

Our last example concerns the structural response of concrete in the spalling test [7]. A compressive pulse is generated by impact on one side of a cylindrical specimen. When the stress wave reaches the other free end, a tensile reflected pulse propagating in the opposite direction is induced. The superposition of incident and reflected signals generates a tensile loading inside the specimen leading to failure.

We consider a one-dimensional model for a bar of length $0.14 \mathrm{~m}$. The material constants for R30A7 concrete and the model parameters are the same as in the previous section. The shape of the compressive pulse is $\sigma(t)=\sigma_{m} \cdot \exp \left(\frac{-(t-0.5 . T)^{2}}{2 S}\right)$ for $t \in(0, T), T=70 \mu s$, 


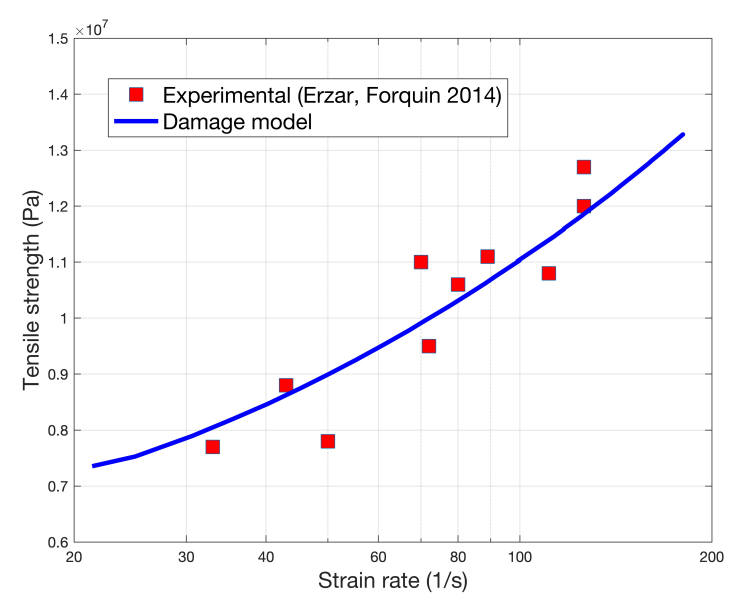

Figure 5 Spalling test on concrete : simulation results and experimental data for the tensile strength vs. the strain rate.

with the amplitude $\sigma_{m}$ taking values in the interval $11-50 M P a$ and the constant $S=1 / 120000$.

The elasto-damage initial and boundary value problem is solved with a finite elements - finite differences integration scheme. Finite elements with linear shape functions are considered for the spatial discretization. We adopted an unconditionally stable implicit Newmark scheme $(\gamma=0.5, \beta=0.25)$ for the time integration of the equation of motion and a fourth order Runge-Kutta integration in time for the damage law. A time step of $1.10^{-8} s$ and uniform finite element meshes with 1000 elements are used. Mesh objectivity has been checked for the numerical simulations.

The strain-rate range of the experimental data [7] is covered by computations with different amplitudes $\sigma_{m}$. The resulting tensile strengths are plotted in Figure 5. They are close to the results of the local response in Figure 3, obtained under the hypothesis of constant strain-rate. They are also in good agreement with the experimental data for the spalling tests.

\section{Conclusions}

A new dynamic damage model has been proposed by incorporating the Grady-Glenn-Chudnovsky fragment size relation in a two-scale damage evolution law. For the given expression of the fragment size, a specific calibration procedure involving a low-dynamics strength and a parameter controlling the degradation of the elastic moduli and characterizing the brittleness of the material response has been specified. Results of local response analysis and 1D finite element simulations of the spalling test were presented. Good correspondence between the tensile strength predictions and experimental data is obtained for concrete and ceramic materials in a large range of dynamic strain-rates.

\section{Références}

1. Atiezo K.M., Chen W., Dascalu C., Loading rate effects on dynamic failure of quasi-brittle solids : Simulations with a two-scale damage model. Theor. Appl. Fract. Mech., 100 : 269-280 (2019).

2. Daphalapurkar, N. P., Ramesh, K. T., Graham-Brady, L., Molinari, J.-F., Predicting variability in the dynamic failure strength of brittle materials considering pre-existing flaws, J. Mech. Phys. Solids, 59(2), pp. 297-319 (2011).

3. Dascalu C., Multiscale modeling of rapid failure in brittle solids : branching instabilities. Mech. Mater. 199 : 27652778 (2018).

4. Dascalu, C., Gbetchi K., Dynamic evolution of damage by microcracking with heat dissipation. Int. J. Solids Struct. 174-175 : 128-144 (2019).

5. Drugan W.J. Dynamic fragmentation of brittle materials : analytical mechanics-based models. J. Mech. Phys. Solids, 49 :1181-1208 (2001).

6. Erzar, B., Ecaillage, cratérisation et comportement en traction dynamique de bétons sous impact : approches expérimentales et modélisation, PhD thesis, Metz, France (2010).

7. Erzar, B., Forquin, P., Analysis and modelling of the cohesion strength of concrete at high strain-rates. Int. J. Solids Struct. 51 : 2559-2574 (2014).

8. Galvez Diaz-Rubio, F., Rodriguez Perez, J., Sanchez Galvez, V., The spalling of long bars as a reliable method of measuring the dynamic tensile strength of ceramics, Int. J. Impact Engng. $27: 161-177$ (2002).

9. Glenn, L.A., Chudnovsky, A., Strain-energy effects on dynamic fragmentation. J. Appl. Phys. 59 :1379-1380 (1986).

10. Grady, D.E., Local inertial effects in dynamic fragmentation. J. Appl. Phys. 53 : 322-325 (1982).

11. Grady, D.E., Length scales and size distributions in dynamic fragmentation. Int. J. Fract., 163 : 85-99 (2010).

12. Hu, G., Liu, J., Graham-Brady, L., Ramesh, K. T., A 3D mechanistic model for brittle materials containing evolving flaw distributions under dynamic multiaxial loading, J. Mech. Phys. Solids 78, pp. 269-297 (2015).

13. Keita, O., Dascalu, C., François, B., A two-scale model for dynamic damage evolution. J. Mech. Phys. Solids, $64: 170-183$ (2014).

14. Kimberley, J., Ramesh, K. T., Daphalapurkar, N. P., A scaling law for the dynamic strength of brittle solids, Acta Materialia, 61(9), pp. 3509-3521 (2013).

15. Levy S., Molinari J.-F., Dynamic fragmentation of ceramics, signature of defects and scaling of fragment sizes, J. Mech. Phys. Solids, 58, pp. 12-26 (2010).

16. Najar, J., Dynamic tensile fracture phenomena at wave propagation in ceramic bars. Journal de Physique IV Colloque, 4 : C8-647-C8-652 (1994).

17. Pham, K., Marigo, J.J., From the onset of damage to rupture : construction of responses with damage localization for a general class of gradient damage models, Cont. Mech. Thermodyn. 25 :147-171 (2013). 
18. Swanson, G.D., Fracture energies of ceramics, J. Amer. Ceram. Soc., 55 : 48-49 (1972).

19. Zhou, F., Molinari, J.F., Ramesh, K.T., Characteristic fragment size distributions in dynamic fragmentation. Appl. Phys. Lett. 88 : 2619181-3 (2006).

20. Zinszner J.L., Erzar B., Forquin P., Buzaud E. Dynamic fragmentation of an alumina ceramic subjected to shockless spalling : An experimental and numerical study. J. Mech. Phys. Solids, 85 :112-127 (2015). 